\title{
Correction to: Contradictions, from Consistency to Inconsistency
}

\author{
Walter Carnielli and Jacek Malinowski
}

\section{Correction to:}

W. Carnielli and J. Malinowski (eds.), Contradictions, from Consistency to Inconsistency, Trends in Logic 47, https://doi.org/10.1007/978-3-319-98797-2

The book was inadvertently published with chapter author's incorrect family name. This information has been updated from "First Name: María and Family Name: del Rosario Martínez-Ordaz" to "First Name: Maria del Rosario and Family Name: Martínez-Ordaz" in the initially published version of Chapters "The Possibility and Fruitfulness of a Debate on the Principle of Non-contradiction" and "Keeping Globally Inconsistent Scientific Theories Locally Consistent". The correction chapters and book have been updated with the changes.

The updated version of these chapters can be found at https://doi.org/10.1007/978-3-319-98797-2_3

https://doi.org/10.1007/978-3-319-98797-2_4 